\title{
On some conjectures concerning critical independent sets of a graph
}

\author{
Taylor Short* \\ Department of Mathematics \\ University of South Carolina \\ shorttm2@mailbox.sc.edu
}

Submitted: Sep 18, 2015; Accepted: May 23, 2016; Published: Jun 10, 2016

Mathematics Subject Classifications: 05C75, 05C69, 05C70

\begin{abstract}
Let $G$ be a simple graph with vertex set $V(G)$. A set $S \subseteq V(G)$ is independent if no two vertices from $S$ are adjacent. For $X \subseteq V(G)$, the difference of $X$ is $d(X)=|X|-|N(X)|$ and an independent set $A$ is critical if $d(A)=\max \{d(X)$ : $X \subseteq V(G)$ is an independent set $\}$ (possibly $A=\emptyset$ ). Let nucleus $(G)$ and $\operatorname{diadem}(G)$ be the intersection and union, respectively, of all maximum size critical independent sets in $G$. In this paper, we will give two new characterizations of König-Egerváry graphs involving nucleus $(G)$ and $\operatorname{diadem}(G)$. We also prove a related lower bound for the independence number of a graph. This work answers several conjectures posed by Jarden, Levit, and Mandrescu.
\end{abstract}

Keywords: maximum independent set; maximum critical independent set; KönigEgerváry graph; maximum matching; core; corona; ker; diadem; nucleus.

\section{Introduction}

In this paper $G$ is a simple graph with vertex set $V(G),|V(G)|=n$, and edge set $E(G)$. The set of neighbors of a vertex $v$ is $N_{G}(v)$ or simply $N(v)$ if there is no possibility of ambiguity. If $X \subseteq V(G)$, then the set of neighbors of $X$ is $N(X)=\cup_{u \in X} N(u), G[X]$ is the subgraph induced by $X$, and $X^{c}$ is the complement of the subset $X$. For sets $A, B \subseteq V(G)$, we use $A \backslash B$ to denote the vertices belonging to $A$ but not $B$. For such disjoint $A$ and $B$ we let $(A, B)$ denote the set of edges such that each edge is incident to both a vertex in $A$ and a vertex in $B$.

A matching $M$ is a set of pairwise non-incident edges of $G$. A matching of maximum cardinality is a maximum matching and $\mu(G)$ is the cardinality of such a maximum

${ }^{*}$ Supported in part by the NSF DMS under contract 1300547. 
matching. For a set $A \subseteq V(G)$ and matching $M$, we say $A$ is saturated by $M$ if every vertex of $A$ is incident to an edge in $M$. For two disjoint sets $A, B \subseteq V(G)$, we say there is a matching $M$ of $A$ into $B$ if $M$ is a matching of $G$ such that every edge of $M$ belongs to $(A, B)$ and each vertex of $A$ is saturated. An $M$-alternating path is a path that alternates between edges in $M$ and those not in $M$. An $M$-augmenting path is an $M$-alternating path which begins and ends with vertices not saturated by $M$.

A set $S \subseteq V(G)$ is independent if no two vertices from $S$ are adjacent. An independent set of maximum cardinality is a maximum independent set and $\alpha(G)$ is the cardinality of such a maximum independent set. For a graph $G$, let $\Omega(G)$ denote the family of all its maximum independent sets, let

$$
\operatorname{core}(G)=\bigcap\{S: S \in \Omega(G)\}, \quad \text { and } \quad \operatorname{corona}(G)=\bigcup\{S: S \in \Omega(G)\} .
$$

See $[1,15]$ for background and properties of core $(G)$ and corona $(G)$.

For a graph $G$ and a set $X \subseteq V(G)$, the difference of $X$ is $d(X)=|X|-|N(X)|$ and the critical difference $d(G)$ is $\max \{d(X): X \subseteq V(G)\}$. Zhang [24] showed that $\max \{d(X): X \subseteq V(G)\}=\max \{d(S): S \subseteq V(G)$ is an independent set $\}$. The set $X$ is a critical set if $d(X)=d(G)$. The set $S \subseteq V(G)$ a critical independent set if $S$ is both a critical set and independent. A critical independent set of maximum cardinality is called a maximum critical independent set. Note that for some graphs the empty set is the only critical independent set, for example odd cycles or complete graphs. See [2,12,13,24] for more background and properties of critical independent sets.

Finding a maximum independent set is a well-known NP-hard problem. Zhang [24] first showed that a critical independent set can be found in polynomial time. Butenko and Trukhanov [2] showed that every critical independent set is contained in a maximum independent set, thereby directly connecting the problem of finding a critical independent set to that of finding a maximum independent set.

For a graph $G$ the inequality $\alpha(G)+\mu(G) \leqslant n$ always holds. A graph $G$ is a KönigEgerváry graph if $\alpha(G)+\mu(G)=n$. According to the classical result of König [10] and Egerváry [4], all bipartite graphs are König-Egerváry graphs. There are non-bipartite graphs which are König-Egerváry as well, see Figure 2 for an example. We adopt the convention that the empty graph $K_{0}$, without vertices, is a König-Egerváry graph.

Deming [3] and Sterboul [22] were the first to give characterizations of König-Egerváry graphs. A matching $M$ of a graph is perfect if every vertex of the graph is saturated by $M$. With respect to a matching $M$, a blossom is an odd cycle where half of one less than the number of edges in the cycle belong to $M$. The unique vertex of the blossom not saturated by $M$ is called the blossom tip. A blossom pair is a pair of blossoms whose tips are joined by an $M$-alternating path with an odd number of edges that begins and ends with edges in $M$. Deming proved that if $G$ is a graph with a perfect matching $M$, then $G$ is a König-Egerváry graph if, and only if, $G$ contains no blossom pair. Sterboul gave an equivalent characterization.

Gavril [7] introduced red/blue-split graphs, a generalization of König-Egerváry graphs and split graphs. A graph is a red/blue-split graph if its edges can be colored using red, blue, or both colors such that the vertices can be partitioned into a red and blue 
independent set (where red or blue independent set is an independent set in the graph made of red or blue edges, respectively). Gavril [6] also proved that given a maximum matching of a graph $G$, the problem of determining whether $G$ is a König-Egerváry graph has complexity $O(n+|E(G)|)$.

Korach et al. [11] described red/blue-split graphs in terms of certain forbidden configurations. This led them to a characterization of König-Egerváry graphs in terms of certain forbidden subgraphs with respect to a maximum matching. Lovász [20] gave a characterization of König-Egerváry graphs having a perfect matching, in terms of certain forbidden subgraphs with respect to a particular perfect matching.

Larson and Pepper [14] gave a partial characterization of König-Egerváry graphs involving the annihilation number of a graph. For a graph $G$ with degree sequence $d_{1} \leqslant d_{2} \leqslant \ldots \leqslant d_{n}$, the annihilation number $a=a(G)$ is the largest index such that $\sum_{i=1}^{a} d_{i} \leqslant|E(G)|$. An annihilating set $A$ is a subset of the vertices such that the sum of the degrees of the vertices in $A$ does not exceed $|E(G)|$. We say that $A$ is a maximum annihilating set if $|A|=a(G)$. Larson and Pepper proved that if $G$ is a graph with $a(G) \geqslant \frac{n}{2}$, then $a(G)=\alpha(G)$ if, and only if, $G$ is a König-Egerváry graph and every maximum independent set is also a maximum annihilating set.

Larson [13] also showed that König-Egerváry graphs are closely related to critical independent sets.

Theorem 1. [13] A graph $G$ is König-Egerváry if, and only if, every maximum independent set in $G$ is critical.

Theorem 2. [13] For any graph $G$, there is a unique set $X \subseteq V(G)$ such that all of the following hold:

(i) $\alpha(G)=\alpha(G[X])+\alpha\left(G\left[X^{c}\right]\right)$,

(ii) $G[X]$ is a König-Egerváry graph,

(iii) for every non-empty independent set $S$ in $G\left[X^{c}\right],|N(S)|>|S|$, and

(iv) for every maximum critical independent set $I$ of $G, X=I \cup N(I)$.

Larson [12] proved that a maximum critical independent set can be found in polynomial time. So the decomposition in Theorem 2 of a graph $G$ into $X$ and $X^{c}$ is also computable in polynomial time. Figure 1 gives an example of this decomposition, where both the sets $X$ and $X^{c}$ are non-empty. Recall, for some graphs the empty set is the only critical independent set, so for such graphs the set $X$ would be empty. If a graph $G$ is a KönigEgerváry graph, then the set $X^{c}$ would be empty. We adopt the convention that if $K_{0}$ is empty graph, then $\alpha\left(K_{0}\right)=0$.

In $[9,17]$ the following concepts were introduced: for a graph $G$,

$$
\begin{aligned}
\operatorname{ker}(G) & =\bigcap\{S: S \text { is a critical independent set in } G\}, \\
\text { diadem }(G) & =\bigcup\{S: S \text { is a critical independent set in } G\}, \text { and } \\
\text { nucleus }(G) & =\bigcap\{S: S \text { is a maximum critical independent set in } G\} .
\end{aligned}
$$

However, the following result due to Larson allows us to use a more suitable definition for $\operatorname{diadem}(G)$. 


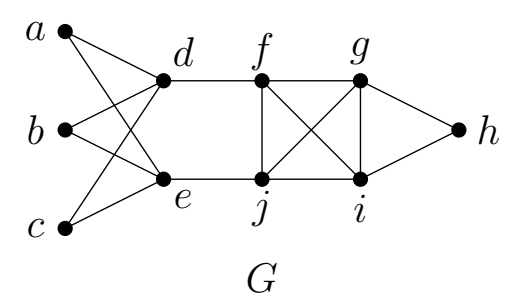

Figure 1: $G$ has maximum critical independent set $I=\{a, b, c\}$. Theorem 2 gives that $X=\{a, b, c, d, e\}$ and $X^{c}=\{f, g, h, i, j\}$.

Theorem 3. [12] Each critical independent set is contained in some maximum critical independent set.

For the remainder of this paper we define

$$
\text { diadem }(G)=\bigcup\{S: S \text { is a maximum critical independent set in } G\} .
$$

Note that if $G$ is a graph where the empty set is the only critical indepedent set (including the case $G=K_{0}$, the empty $\left.\operatorname{graph}\right)$, then $\operatorname{ker}(G), \operatorname{diadem}(G)$, and $\operatorname{nucleus}(G)$ are all empty. See Figure 2 for examples of the sets $\operatorname{ker}(G)$, $\operatorname{diadem}(G)$, and nucleus $(G)$.

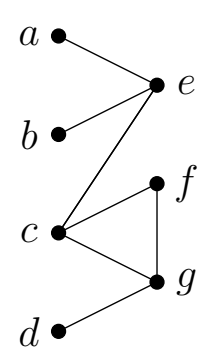

$G_{1}$

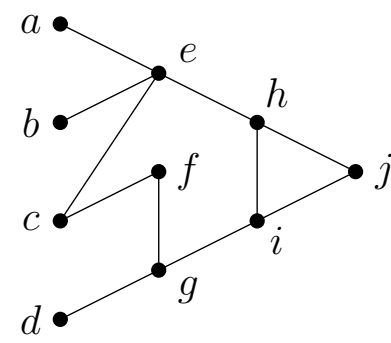

$G_{2}$

Figure 2: $G_{1}$ is a König-Egerváry graph with $\operatorname{ker}\left(G_{1}\right)=\{a, b\} \subsetneq \operatorname{core}\left(G_{1}\right)=$ $\operatorname{nucleus}\left(G_{1}\right)=\{a, b, d\}$ and $\operatorname{diadem}\left(G_{1}\right)=\operatorname{corona}\left(G_{1}\right)=\{a, b, c, d, f\} . G_{2}$ is not a König-Egerváry graph and has $\operatorname{ker}\left(G_{2}\right)=\operatorname{core}\left(G_{2}\right)=\{a, b\} \subsetneq \operatorname{nucleus}\left(G_{2}\right)=\{a, b, d\}$ and $\operatorname{diadem}\left(G_{2}\right)=\{a, b, c, d, f\} \subsetneq \operatorname{corona}(G)=\{a, b, c, d, f, g, h, i, j\}$.

In $[8,9]$, the following necessary conditions for König-Egerváry graphs were given:

Theorem 4. [8] If $G$ is a König-Egerváry graph, then

(i) $\operatorname{diadem}(G)=\operatorname{corona}(G)$, and

(ii) $|\operatorname{ker}(G)|+|\operatorname{diadem}(G)| \leqslant 2 \alpha(G)$.

Theorem 5. [9] If $G$ is a König-Egerváry graph, then $|\operatorname{nucleus}(G)|+|\operatorname{diadem}(G)|=$ $2 \alpha(G)$. 
In [8] it was conjectured that condition $(i)$ of Theorem 4 is sufficient for König-Egerváry graphs and in [9] it was conjectured the necessary condition in Theorem 5 is also sufficient. The purpose of this paper is to affirm these conjectures by proving the following new characterizations of König-Egerváry graphs.

Theorem 6. For a graph $G$, the following are equivalent:

(i) $G$ is a König-Egerváry graph,

(ii) $\operatorname{diadem}(G)=\operatorname{corona}(G)$, and

(iii) $|\operatorname{diadem}(G)|+|\operatorname{nucleus}(G)|=2 \alpha(G)$.

The paper [8] gives an upper bound for $\alpha(G)$ in terms of unions and intersections of maximum independent sets, proving

$$
2 \alpha(G) \leqslant|\operatorname{core}(G)|+|\operatorname{corona}(G)|
$$

for any graph $G$. It is natural to ask whether a similar lower bound for $\alpha(G)$ can be formulated in terms of unions and intersections of critical independent sets. Jarden, Levit, and Mandrescu in [8] conjectured that for any graph $G$, the inequality $|\operatorname{ker}(G)|+$ $|\operatorname{diadem}(G)| \leqslant 2 \alpha(G)$ always holds. We will prove a slightly stronger statement. By Theorem 3 we see that $\operatorname{ker}(G) \subseteq \operatorname{nucleus}(G)$ holds implying that $|\operatorname{ker}(G)|+|\operatorname{diadem}(G)| \leqslant$ $\mid$ nucleus $(G)|+| \operatorname{diadem}(G) \mid$. In section 4 we will prove the following statement, resolving the cited conjecture:

Theorem 7. For any graph $G$,

$$
|\operatorname{nucleus}(G)|+|\operatorname{diadem}(G)| \leqslant 2 \alpha(G) .
$$

It would be interesting to know whether the sets nucleus $(G)$ and $\operatorname{diadem}(G)$, or their sizes, can be computed in polynomial time.

\section{Some structural lemmas}

Here we prove several crucial lemmas which will be needed in our proofs. Our results hinge upon the structure of the set $X$ as described in Theorem 2 .

Lemma 8. Let $I$ be a maximum critical independent set in $G$ and set $X=I \cup N(I)$. Then $\operatorname{diadem}(G) \cup N(\operatorname{diadem}(G))=X$.

Proof. By Theorem 2 the set $X$ is unique in $G$, that is, for any maximum critical independent set $S, X=S \cup N(S)$. Then $\operatorname{diadem}(G) \cup N(\operatorname{diadem}(G))=X$ follows by definition.

Lemma 9. Let $I$ be a maximum critical independent set in $G$ and set $X=I \cup N(I)$. Then $\operatorname{diadem}(G) \subseteq \operatorname{diadem}(G[X])$ and nucleus $(G[X]) \subseteq \operatorname{nucleus}(G)$. 
Proof. Let $S$ be a maximum critical independent set in $G$. Using Theorem 2 we see that $S$ is a maximum independent set in $G[X]$ and also $G[X]$ is a König-Egerváry graph. Then Theorem 1 gives that $S$ must also be critical in $G[X]$, which implies that $\operatorname{diadem}(G) \subseteq$ $\operatorname{diadem}(G[X])$.

Now let $v \in \operatorname{nucleus}(G[X])$. Then $v$ belongs to every maximum critical indepedent set in $G[X]$. As remarked above, since every maximum critical independent set in $G$ is also a maximum critical independent set in $G[X]$, then $v$ belongs to every maximum critical independent set in $G$. This shows that $v \in \operatorname{nucleus}(G)$ and nucleus $(G[X]) \subseteq \operatorname{nucleus}(G)$ follows.

Lemma 10. Suppose $I$ is a non-empty maximum critical independent set in $G$, set $X=$ $I \cup N(I)$, let $A=\operatorname{nucleus}(G) \backslash \operatorname{nucleus}(G[X])$, and let $S$ be a maximum independent set in $G[X]$. For $S^{\prime} \subseteq S \cap N(A)$, if there exists $A^{\prime} \subseteq A$ such that $N\left(A^{\prime}\right) \cap S \subseteq S^{\prime}$, then $\left|S^{\prime}\right| \geqslant\left|A^{\prime}\right|$.

Proof. For $S^{\prime} \subseteq S \cap N(A)$ suppose such an $A^{\prime}$ exists. For sake of contradiction, suppose that $\left|S^{\prime}\right|<\left|A^{\prime}\right|$. Since $A^{\prime} \subseteq \operatorname{nucleus}(G)$, then $A^{\prime}$ is an independent set. Also since $A^{\prime} \subseteq \operatorname{nucleus}(G) \subseteq \operatorname{diadem}(G)$, by Lemma 8 we have $A^{\prime} \subseteq X$. Furthermore, since $N\left(A^{\prime}\right) \cap S \subseteq S^{\prime}$ then $A^{\prime} \cup\left(S \backslash S^{\prime}\right)$ is an independent set in $G[X]$. Now by assumption $\left|S^{\prime}\right|<\left|A^{\prime}\right|$, so $A^{\prime} \cup\left(S \backslash S^{\prime}\right)$ is an independent set in $G[X]$ larger than $S$, which cannot happen. Therefore we must have $\left|S^{\prime}\right| \geqslant\left|A^{\prime}\right|$ as desired.

Lemma 11. Let $I$ be a maximum critical independent set in $G$ and set $X=I \cup N(I)$. Then

$$
|\operatorname{nucleus}(G)|+|\operatorname{diadem}(G)| \leqslant|\operatorname{nucleus}(G[X])|+|\operatorname{diadem}(G[X])| .
$$

Proof. First note that if the set $X$ is empty, then by Lemma 8 both sides of the inequality are zero. So let us assume that $X$ is non-empty. Now consider the set $A=\operatorname{nucleus}(G) \backslash$ $\operatorname{nucleus}(G[X])$. If this independent set is empty, then nucleus $(G)=\operatorname{nucleus}(G[X])$ and there is nothing to prove since $\operatorname{diadem}(G) \subseteq \operatorname{diadem}(G[X])$ holds by Lemma 9 . If $A$ is non-empty, for each $v \in A$ there is some maximum independent set $S$ of $G[X]$ which doesn't contain $v$. Since $S$ is a maximum independent set there exists $u \in N(v) \cap S$. Since $v \in \operatorname{nucleus}(G)$, then $u$ does not belong to any maximum critical independent set in $G$. Recall by Theorem 2 (ii) $G[X]$ is a König-Egerváry graph, so Theorem 1 gives that $S$ is a maximum critical independent set in $G[X]$. It follows that $u \in$ $\operatorname{diadem}(G[X]) \backslash \operatorname{diadem}(G)$, which shows each vertex in $A$ is adjacent to at least one vertex in $\operatorname{diadem}(G[X]) \backslash \operatorname{diadem}(G)$.

Now we will show there is a maximum matching from $A$ into $\operatorname{diadem}(G[X]) \backslash \operatorname{diadem}(G)$ with size $|A|$. For sake of contradiction, suppose such a matching $M$ has less than $|A|$ edges. Then there exists some vertex $v \in A$ not saturated by $M$. By the above, $v$ is adjacent to some vertex $u \in \operatorname{diadem}(G[X]) \backslash \operatorname{diadem}(G)$. Since $M$ is maximum, $u$ is matched to some vertex $w \in A$ under $M$. Now let $S$ be a maximum independent set of $G[X]$ containing $u$. We now restrict ourselves to the subgraph induced by the edges $(A \cap N(S), S \cap N(A))$, noting this subgraph is bipartite since both $A \cap N(S)$ and $S \cap N(A)$ are independent. In this subgraph, consider the set $\mathcal{P}$ of all $M$-alternating paths starting 
with the edge $v u$. Note that all such paths must start with the vertices $v, u$, then $w$. Also, such paths must end at either a matched vertex in $A \cap N(S)$ or an unmatched vertex in $S \cap N(A)$.

We wish to show that there is some alternating path ending at an unmatched vertex in $S \cap N(A)$. For sake of contradiction, suppose all alternating paths end at a matched vertex in $A \cap N(S)$ and let $V(\mathcal{P})$ denote the union of all vertices belonging to such an alternating path. We aim to show this scenario contradicts Lemma 10. Now clearly we must have $N(V(\mathcal{P}) \cap A) \cap S \subseteq V(\mathcal{P}) \cap S$, else we could extend an alternating path to any vertex in $(N(V(\mathcal{P}) \cap A) \cap S) \backslash(V(\mathcal{P}) \cap S)$. Also, since all paths in $\mathcal{P}$ end at a matched vertex in $A \cap N(S)$, then every vertex of $V(\mathcal{P}) \cap S$ is matched under $M$, and such a situation should look as in Figure 3.

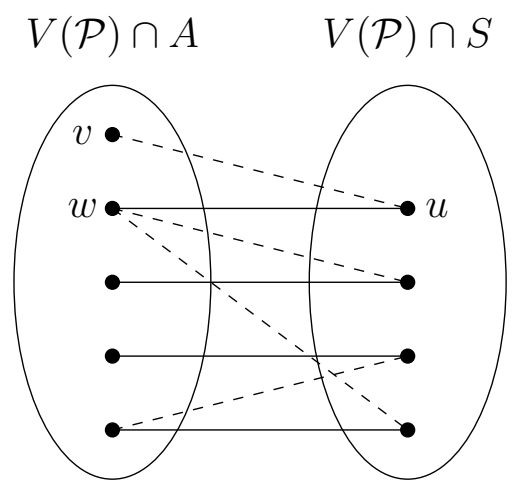

Figure 3: What the $M$-alternating paths could look like between $V(\mathcal{P}) \cap A$ and $V(\mathcal{P}) \cap S$, where solid lines represent matched edges in $M$ and dotted lines represent the unmatched edges.

From this it follows that $|V(\mathcal{P}) \cap S|<|V(\mathcal{P}) \cap A|$. The previous statements exactly contradict Lemma 10, so there is some alternating path $P$ ending at an unmatched vertex $x \in S \cap N(A)$. This means that $P$ is an $M$-augmenting path. A well-known theorem in graph theory states that a matching is maximum in $G$ if, and only if, there is no augmenting path [23]. So $P$ being an $M$-augmenting path contradicts our assumption that $M$ is a maximum matching.

Therefore there is a matching $M$ from $A$ into diadem $(G[X]) \backslash \operatorname{diadem}(G)$. This matching implies that $|\operatorname{nucleus}(G) \backslash \operatorname{nucleus}(G[X])| \leqslant|\operatorname{diadem}(G[X]) \backslash \operatorname{diadem}(G)|$. Since both nucleus $(G[X]) \subseteq \operatorname{nucleus}(G)$ and $\operatorname{diadem}(G) \subseteq \operatorname{diadem}(G[X])$ by Lemma 9 , the lemma follows.

\section{New characterizations of König-Egerváry graphs}

Proof (of Theorem 6). First we prove $(i i) \Rightarrow(i)$. Suppose that $\operatorname{diadem}(G)=\operatorname{corona}(G)$ holds and let $I$ be a maximum critical independent set with $X=I \cup N(I)$. We will use the decomposition in Theorem 2 to show that $X^{c}$ must be empty and hence, $G=G[X]$ 
is a König-Egerváry graph. By Lemma 8 we have corona $(G)=\operatorname{diadem}(G) \subseteq X$, in other words every maximum independent set in $G$ is contained in $X$. This implies that $|I|=\alpha(G[X])=\alpha(G)$. Now by Theorem $2(i), \alpha(G)=\alpha(G[X])+\alpha\left(G\left[X^{c}\right]\right)$ showing that we must have $\alpha\left(G\left[X^{c}\right]\right)=0$. Now clearly the result follows, since $\alpha\left(G\left[X^{c}\right]\right)=0$ implies that $X^{c}$ must be empty.

To prove $(i i i) \Rightarrow(i)$, again we will use the decomposition in Theorem 2 to show that $X^{c}$ must be empty and hence, $G$ is a König-Egerváry graph. So suppose that $|\operatorname{diadem}(G)|+|\operatorname{nucleus}(G)|=2 \alpha(G)$ and let $I$ be a maximum critical independent set in $G$ with $X=I \cup N(I)$. Lemma 11 implies that

$$
2 \alpha(G)=|\operatorname{diadem}(G)|+|\operatorname{nucleus}(G)| \leqslant|\operatorname{diadem}(G[X])|+|\operatorname{nucleus}(G[X])| .
$$

Theorem $2(i i)$ gives that $G[X]$ is König-Egerváry, so by Corollary 5 we have

$$
|\operatorname{diadem}(G[X])|+|\operatorname{nucleus}(G[X])|=2 \alpha(G[X])
$$

implying that $\alpha(G) \leqslant \alpha(G[X])$. It follows by Theorem $2(i)$ we must have $\alpha(G)=$ $\alpha(G[X])$, so again we know that $\alpha\left(G\left[X^{c}\right]\right)=0$ which finishes this part of the proof.

The implications $(i) \Rightarrow(i i)$ and $(i) \Rightarrow($ iii $)$ are given in Theorem 4 and in Theorem 5 .

\section{A bound on $\alpha(G)$}

Proof (of Theorem 7). Let $I$ be a maximum critical independent set in $G$ and $X=I \cup$ $N(I)$. By Theorem $2(i i), G[X]$ is a König-Egerváry graph so by Theorem 5 we have

$$
|\operatorname{nucleus}(G[X])|+|\operatorname{diadem}(G[X])|=2 \alpha(G[X]) \leqslant 2 \alpha(G) \text {. }
$$

Now by Lemma 11 we must have

$$
|\operatorname{nucleus}(G)|+|\operatorname{diadem}(G)| \leqslant|\operatorname{nucleus}(G[X])|+|\operatorname{diadem}(G[X])|
$$

and the theorem follows.

Combining Theorem 7 and the inequality $2 \alpha(G) \leqslant|\operatorname{core}(G)|+|\operatorname{corona}(G)|$ proven in [8], the following corollary is immediate.

Corollary 12. For any graph $G$,

$$
|\operatorname{nucleus}(G)|+|\operatorname{diadem}(G)| \leqslant 2 \alpha(G) \leqslant|\operatorname{core}(G)|+|\operatorname{corona}(G)| .
$$

These upper and lower bounds are quite interesting. The fact that every critical independent set is contained in a maximum independent set implies that $\operatorname{diadem}(G) \subseteq \operatorname{corona}(G)$ for all graphs $G$. However, the graph $G_{2}$ in Figure 2 has core $\left(G_{2}\right) \subsetneq$ nucleus $\left(G_{2}\right)$ while the graph $G$ in Figure 1 has nucleus $(G)=\{a, b, c\} \subsetneq \operatorname{core}(G)=\{a, b, c, h\}$. 


\section{Acknowledgements}

Many thanks to my advisor László Székely for feedback on the initial versions of this manuscript. Partial support from the NSF DMS under contract 1300547 is gratefully acknowledged.

\section{References}

[1] E. Boros, M. C. Golumbic, and V. E. Levit, On the number of vertices belonging to all maximum stable sets of a graph, Discrete Applied Mathematics 124 (2002), 17-25.

[2] S. Butenko and S. Trukhanov, Using critical sets to solve the maximum independent set problem, Operations Research Letters 35 (2007), 519-524.

[3] R. W. Deming, Independence Number of Graphs - an Extension of the König-Egerváry Theorem, Discrete Mathematics 27 (1979), 23-33.

[4] E. Egerváry, On combinatorial properties of matrices, Matematikai Lapok 38 (1931), $16-28$.

[5] M. Garey and D. Johnson, Computers and Intractability, W. H. Freeman and Company, New York, 1979.

[6] F. Gavril, Testing for equality between maximum matching and minimum node covering, Inf. Process. Lett. 6 (1977), no. 6, 199-202.

[7] F. Gavril, An efficient solvable graph partition problem to which many problems are reducible, Information Processing Letters 45 (1993), no. 285-290.

[8] A. Jarden, V. E. Levit, and E. Mandrescu, Critical and Maximum Independent Sets of a Graph, arXiv:1506.00255 (2015), 12 pp.

[9] A. Jarden, V. E. Levit, and E. Mandrescu, Monotonic Properties of Collections of Maximum Independent Sets of a Graph, arXiv: 1506.00249 (2015), 15 pp.

[10] D. König, Graphen und Matrizen, Matematikai Lapok 38 (1931), 116-119.

[11] E. Korach, T. Nguyen, and B. Peis, Subgraph characterization of red/blue-split graphs and König-Egerváry graphs, Proceedings of the seventeenth annual acm-siam symposium on discrete algorithms, 2006, pp. 842-850.

[12] C. E. Larson, A Note on Critical Independence Reductions, Bulletin of the Institute of Combinatorics and its Applications 51 (2007), 34-46.

[13] C. E. Larson, The critical independence number and an independence decomposition, European Journal of Combinatorics 32 (2011), 294-300.

[14] C. E. Larson and R. Pepper, Graphs with equal independence and annihilation numbers, The Electronic Journal of Combinatorics 18 (2011), no. 1, \#P180.

[15] V. E. Levit and E. Mandrescu, Combinatorial properties of the family of maximum stable sets of a graph, Discrete Applied Mathematics 117 (2002), 149-161. 
[16] V. E. Levit and E. Mandrescu, On $\alpha^{+}$-stable König-Egerváry graphs, Discrete Mathematics 263 (2003), 179-190.

[17] V. E. Levit and E. Mandrescu, Vertices belonging to all critical independent sets of a graph, SIAM Journal on Discrete Mathematics 26 (2012), 399-403.

[18] V. E. Levit and E. Mandrescu, On maximum matchings in König-Egerváry graphs, Discrete Applied Mathematics 161 (2013), 1635-1638.

[19] V. E. Levit and E. Mandrescu, A set and collection lemma, The Electronic Journal of Combinatorics 21 (2014), no. P1.40.

[20] L. Lovász, Ear-decompositions of matching-covered graphs, Combinatorica 3 (1983), $105-118$.

[21] T. Short, KE theory $\&$ the number of vertices belonging to all maximum independent sets in a graph, Master's Thesis, http://scholarscompass.vcu.edu/etd/2353/, 2011.

[22] F. Sterboul, A characterization of the graphs in which transversal number equals the matching number, Journal of Combinatorial Theory Series B 27 (1979), no. 228-229.

[23] D. West, Introduction to Graph Theory, 2nd ed., Prentice Hall, Inc., Upper Saddle River, NJ, 2001.

[24] C. Q. Zhang, Finding critical independent sets and crtitical vertex subsets are polynomial problems, SIAM Journal on Discrete Mathematics 3 (1990), 431-438. 